\title{
Discovery of a nearby young brown dwarf binary candidate
}

\author{
A. Reiners ${ }^{1, \star}$, A. Seifahrt ${ }^{2}$, and S. Dreizler ${ }^{1}$ \\ 1 Universität Göttingen, Institut für Astrophysik, Friedrich-Hund-Platz 1, 37077 Göttingen, Germany \\ e-mail: Ansgar. Reiners@phys. uni-goettingen.de \\ 2 Department of Physics, University of California, One Shields Avenue, Davis, CA 95616, USA \\ Received 5 February 2010 / Accepted 5 March 2010
}

\section{ABSTRACT}

\begin{abstract}
In near-infrared NaCo observations of the young brown dwarf 2MASS J0041353-562112, we discovered a companion a little less than a magnitude fainter than the primary. The binary candidate has a separation of 143 mas, and the spectral types of the two components are M 6.5 and M 9.0. Colors and flux ratios of the components are consistent with their locations being at the same distance minimizing the probability of the secondary being a background object. The brown dwarf is known to exhibit Li absorption constraining the age to be younger than $\sim 200 \mathrm{Myr}$, and has been suspected of experiencing ongoing accretion, which implies an age as young as $\sim 10 \mathrm{Myr}$. We estimate distance and orbital parameters of the binary as a function of age. For an age of $10 \mathrm{Myr}$, the distance to the system is $50 \mathrm{pc}$, the orbital period is $126 \mathrm{yr}$, and the masses of the components are $\sim 30$ and $\sim 15 M_{\text {Jup }}$. The binary brown dwarf fills a so far unoccupied region in the parameters mass and age; it is a valuable new benchmark object for brown dwarf atmospheric and evolutionary models.
\end{abstract}

Key words. stars: pre-main-sequence - stars: formation - stars: individual: 2MASS J0041353-562112 - stars: low-mass brown dwarfs

\section{Introduction}

Very low mass binaries are of particular interest for a number of reasons. First, at very low masses, the binary fraction and the orbital properties of the binaries carry important information about the way binaries form (e.g., Close et al. 2003; Burgasser et al. 2007). Second, all components of a multiple system share the same evolutionary history so that a comparison between binary components is free of a number of degeneracies. Finally, binaries offer a model-independent way of determining the mass by means of measuring their orbital period. This third point is most important for our understanding of the evolution of low-mass stars and brown dwarfs in particular at young ages, where models still have relatively large uncertainties.

The number of very low mass binaries that have been detected is now substantial. An updated list of binaries with total masses below $0.2 M_{\odot}$ can be found at the Very-Low-Mass Binary Archive ${ }^{1}$, and contains 99 entries as of Jan. 2010. Of particular importance are brown dwarf binaries with independent age constraints because they provide empirical constraints on brown dwarf evolution models. Furthermore, to determine the mass from orbital motion on reasonable timescales, the orbital period should be short enough, and the binaries should not be too far away so that spectroscopic investigation is possible. Usually, young binaries are members of star-forming regions that are located at a distance of $100 \mathrm{pc}$ or more, which makes a detailed investigation of low-mass members very difficult. Therefore, young, nearby, low-mass systems are of very great value for our understanding of low-mass star and brown dwarf evolution.

In this paper, we present the discovery of a new very low mass binary, 2MASS 0041353-562112 (or DENIS-P J0041353562112; Phan Bao et al. 2001, hereafter 2M0041), which is

\footnotetext{
^ Emmy Noether Fellow.

${ }^{1}$ http://www.vlmbinaries.org
}

nearby and probably very young. The age of $2 \mathrm{M} 0041$ is constrained to be lower than $\sim 200 \mathrm{Myr}$ by the detection of $\mathrm{Li}$ in an optical spectrum (Reiners \& Basri 2009). Reiners (2009) presents evidence of accretion deduced from the intensity and shape of emission lines, in particular $\mathrm{H} \alpha$. Ongoing accretion would suggest that 2M0041 may even be as young as $10 \mathrm{Myr}$. Space motion of $2 \mathrm{M} 0041$ is consistent with it being a member of the $\sim 20$ Myr old Tuc-Hor association or the $\sim 12$ Myr old $\beta$ Pic association. Unfortunately, the distance to $2 \mathrm{M} 0041$ is not yet known because no parallax measurement has so far been available. The distance from spectrophotometry would be $17 \mathrm{pc}$ if the object was an old, single field star, but the real distance is of course larger because the young object is more luminous. As long as no parallax is measured for 2M0041, age, mass, and distance are free parameters that can be constrained by measuring the orbital period of the system.

\section{Data and analysis}

Data were obtained with $\mathrm{NaCo}$, the Nasmyth Adaptive Optics System (NAOS) and Near-Infrared Imager and Spectrograph (CONICA) at ESO's Very Large Telescope (Lenzen et al. 2003; Rousset et al. 2003). Four images were obtained in service mode on August 14, 2009: one image was taken in $J$, one in $H$, and two images were obtained in $K_{\mathrm{s}}$. We obtained ten individual frames, each of them being the average of 3 observations with an individual exposure time of $30 \mathrm{~s}$ each. Thus, the total exposure time is $15 \mathrm{~min}$ per image. Individual images were jittered using a $5^{\prime \prime}$ jitter box, this allowed efficient reduction of the sky background. The adaptive optics system was used with the N90C10 dichroic using 90\% of the light for AO and only $10 \%$ for the science camera. We chose this option because no bright AO source was available nearby. All observations were carried out at low airmass $(<1.3)$ and good seeing conditions $\left(<0.8^{\prime \prime}\right)$. We used the S13 camera with a field of view of $14^{\prime \prime} \times 14^{\prime \prime}$ and 


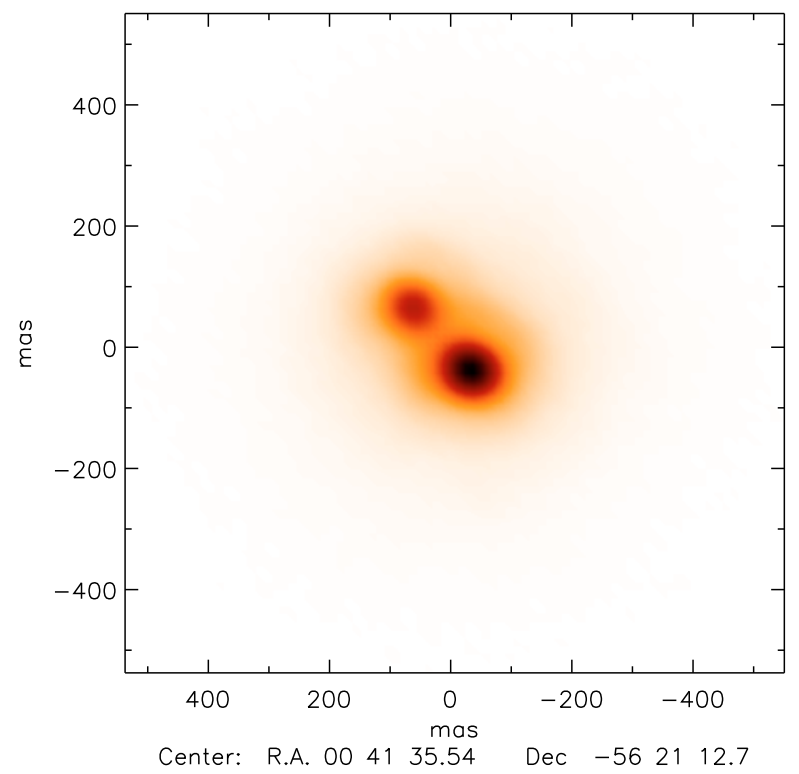

Fig. 1. NACO $K$-band image of 2M0041, North is up and East is left. Axis labels denote coordinates in mas relative to the center of the image.

a pixel scale of 13.2 mas/pix. For our analysis, we use the standard pipeline products that are provided for service mode observations. Data reduction includes dark subtraction, flat fielding, and sky subtraction using the jittered images. A close-up of one of the two $K_{\mathrm{s}}$-band images is shown in Fig. 1. We clearly resolve $2 \mathrm{M} 0041$ as a binary with two components that differ somewhat in brightness; the component to the SW appears brighter than the NE component.

To measure the separation and the flux ratio of the two components, we estimate the PSF from the science images in an iterative procedure. We start with an image of a standard star. We use the so-called zeropoint images that are routinely taken for NaCo service observations. With this first guess of the PSF, we search for stars in our science images and determine their position and flux using the public domain IDL package StarFinder (Diolaiti et al. 2000). Next, we extract the PSF from the science image obtaining information about the flux and position using the routine psf_extract from the StarFinder package. In the next step, we use this PSF to iteratively search for the two components and redetermine the shape of the PSF.

The deduction of the PSF from the science image has the advantage that we do not have to rely on a PSF that was taken at a different time, direction, and object brightness. The shape of the PSF depends sensitively on the adaptive optics performance, which in turn depends on the brightness of the reference target. On the other hand, the separation of the two components that we wish to distinguish is not much larger than the width of the PSF, particularly in the $J$-band. When determining the PSF, this may cause the algorithm to construct a PSF consisting of both components. To avoid this problem, we assume that the PSF has an axisymmetric shape. After each PSF determination, we construct a rotationally averaged version of the PSF that we use in the next iteration of StarFinder.

We successfully identified the two components in all four images using the procedure described above. As a result, we obtain the positions and individual fluxes of the two components, and the PSF of each image.

We show the separation of the two components, their flux ratio, and the width of the PSF from our four images in Table 1. The results of the separation are consistent with each other. The
Table 1. Separation and flux measurements in the four images.

\begin{tabular}{cccccc}
\hline \hline$\#$ & Band & $\begin{array}{c}\text { Separation } \\
{[\mathrm{mas}]}\end{array}$ & PA & Flux ratio & $\begin{array}{c}\text { PSF width } \\
{[\mathrm{mas}]}\end{array}$ \\
\hline 1 & $J$ & 142.3 & $44.90^{\circ}$ & $2.19 \pm 0.11$ & 101.1 \\
2 & $H$ & 142.4 & $43.34^{\circ}$ & $2.06 \pm 0.06$ & 72.3 \\
3 & $K_{\mathrm{s}}$ & 143.0 & $43.46^{\circ}$ & $1.88 \pm 0.05$ & 48.2 \\
4 & $K_{\mathrm{s}}$ & 143.4 & $43.25^{\circ}$ & $1.88 \pm 0.05$ & 52.9 \\
\hline
\end{tabular}

flux ratios of the two $K_{\mathrm{s}}$-band images agree very well, and the flux ratio seems to be a function of color, which is consistent with two components of different temperature. The width of the PSF also varies with wavelength, as expected because the Strehl ratio is lower at shorter wavelengths (Strehl ratios are 0.50 in $K_{\mathrm{s}}$, 0.33 in $H$, and 0.18 in $J$ ). In the $K_{\mathrm{s}}$-band, we note that the width of the PSF is on the order of the diffraction limit. Going to even longer wavelengths would not lead to higher image quality because the diffraction limit increases with wavelength (for example, it is $\sim 100$ mas at $L^{\prime}$ ). In the $J$-band, the two components are not separated well. We tried to use a PSF consisting of different elliptical components, but did not succeed in reproducing our results better than when using our procedure described above. The $J$-band position angle differs from the $H$ - and $K_{\mathrm{s}}$-band results by about 1.5 degree, which may be caused by the slightly irregular shape of the $J$-band PSF. In particular, irregularities in the wings of the $J$-band PSF may lead to a small shift in the suspected stars' positions (here, $\lesssim 0.2$ pix or $\sim 2.5 \%$ of the $J$-band $F W H M$ ). This probably leads to a slight dependence of the photocenter position on the stars' intensity in the $J$-band.

To quantify the uncertainty in our measurements, we performed Monte Carlo simulations for all three bands. In each band, we constructed a series of 1000 images of a binary consisting of two objects with different flux ratios, separations, and position angles. We uniformly varied these values about those found in the real data, and used asymmetric PSFs consisting of two elliptical Gaussian components that scatter about a description optimized to fit the rotationally symmetric PSF from the procedure described above. Noise was added to the artificial data to match the quality of the real images. In the $H$ - and $K$-bands, the fitting process turned out to be very stable. The clear separation of the two components because of the narrow PSF allows a robust fitting process even for an asymmetric PSF. We derived the uncertainties in the flux ratio from the scatter about the mean of our simulations. The $2 \sigma$-scatter in the flux ratio is $3 \%$ for $H$ and $2 \%$ for $K_{\mathrm{s}}$. The $J$-band uncertainties are larger, not only because of the larger FWHM of the PSF but also because of the asymmetry in the PSF mentioned above. Thus, the fitting process in the $J$-band image depends sensitively on assumptions about the shape of the PSF. Because deconvolving that shape is less reliable in the $J$-band image, we decided not to simply adopt the $J$-band uncertainties inferred by our Monte-Carlo approach, but conservatively estimate them to be roughly a factor of two larger because of PSF-dependent systematics. We adopt a $J$-band flux ratio uncertainty of $5 \%$, which agrees with the scatter that we found during our attempts using different PSF assumptions.

\section{Photometry of the components}

Photometric measurements of $2 \mathrm{M} 0041$ in $J, H$, and $K_{\mathrm{S}}$ are available from the Two Micron All Sky Survey (2MASS, Skrutskie et al. 2006). The 2MASS magnitude of $2 \mathrm{M} 0041$ reflects the combined flux of both components. With the NaCo observations, we can now separate the combined flux into two components 
Table 2. Measured parameters of the system.

\begin{tabular}{cccc}
\hline \hline & 2MASS & \multicolumn{2}{c}{ Individual photometry } \\
Parameter & $\mathrm{A}+\mathrm{B}$ & $\mathrm{A}$ & $\mathrm{B}$ \\
\hline$J$ & $11.96 \pm 0.02$ & $12.37 \pm 0.03$ & $13.22 \pm 0.04$ \\
$H$ & $11.32 \pm 0.02$ & $11.75 \pm 0.02$ & $12.53 \pm 0.03$ \\
$K_{\mathrm{S}}$ & $10.86 \pm 0.03$ & $11.32 \pm 0.03$ & $12.01 \pm 0.03$ \\
$J-K_{\mathrm{s}}$ & $1.10 \pm 0.04$ & $1.05 \pm 0.04$ & $1.21 \pm 0.05$ \\
$\Delta J$ & & $0.85 \pm 0.04$ \\
$\Delta H$ & & $0.78 \pm 0.02$ \\
$\Delta K_{\mathrm{s}}$ & & $0.69 \pm 0.02$ \\
SpT & & $\mathrm{M} 6.5 \pm 1$ & $\mathrm{M} 9.0 \pm 1$ \\
Position Angle & & \multicolumn{2}{c}{$43.6^{\circ} \pm 0.6^{\circ}$} \\
Separation [mas] & & \multicolumn{2}{c}{$142.8 \pm 0.5$} \\
\hline
\end{tabular}

using the 2MASS magnitudes and the flux ratios $f_{1} / f_{2}$ in the three filters, for the $J$-band obtaining

$J_{2}=J+2.5 \log \left[1+\left(\frac{f_{1}}{f_{2}}\right)_{J}\right]$.

The results for individual $J, H$, and $K_{\mathrm{s}}$ magnitudes are summarized in Table 2. Based on the assumption that the two components form a binary (i.e., are both located at the same distance $d$ ), we can infer the individual spectral types from both the magnitude differences and the information about the combined spectral type provided by the integrated light. Correlations between the absolute $J$-magnitude and spectral types were reported by Dahn et al. (2002) and Cruz et al. (2003). The combined spectral type of $2 \mathrm{M} 0041$ is M 7.5 with an uncertainty of \pm 0.5 (Phan Bao \& Bessel 2006). Using the linear relation from Dahn et al.(we find that the spectral type difference between the two individual components is 2.5 spectral classes. The dispersion of objects about the relation from Dahn et al. is $0.25 \mathrm{mag}$, which translates into an individual uncertainty of 0.7 spectral classes for individual objects, and an uncertainty of 1.0 spectral classes for the difference between two objects. This dispersion is much larger than the uncertainty caused by the photometric differences of 2M0041 A and $\mathrm{B}$ and we can neglect the latter.

The linear relation between spectral type and absolute magnitude enables us to use the combined spectral type (M 7.5) to determine the spectral type range of $2 \mathrm{M} 0041 \mathrm{~A}$ and B. Using flux ratio and individual magnitudes, we can construct an artificial average magnitude of the combined system $2 \mathrm{M} 0041 \mathrm{AB}$ $\left(\tilde{M}_{J, \mathrm{AB}}=12.63, \tilde{M}_{K, \mathrm{AB}}=11.55\right)$, which in this system must coincide with spectral type M 7.5 \pm 0.5 . From this, we derive individual spectral types for 2M0041 A and B of M $6.5 \pm 1.0$ and M9.0 \pm 1.0 , respectively.

An independent check of our assumption that both components are located at the same distance can be make by examining the colors of $2 \mathrm{M} 0041 \mathrm{~A}$ and B. The typical $J-K$ colors of late-M objects are given, e.g., by Hawley et al. (2002), Dahn et al. (2002), and West et al. (2008). The colors of 2M0041 A are indicative of a spectral type in the range M 5-M9, while $2 \mathrm{M} 0041 \mathrm{~B}$ falls in the range M 8.5-early L. Thus, the colors and flux ratios of $2 \mathrm{M} 0041 \mathrm{~A}$ and $\mathrm{B}$ are consistent with the assumption that both components belong to a binary system. Given a dispersion of $0.2 \mathrm{mag}$ in the $J-K$ relation and much smaller uncertainties in our measurements of flux ratios and colors, the difference in distance modulus between the two components is unlikely to be greater than $6 \mathrm{pc}$.

Table 2 also indicates the mean position angle and separation. For the position angle, the individual measurements are weighted according to the inverse PSF widths of the images to obtain $43.6^{\circ} \pm 0.6^{\circ}$. The mean separation calculated from the four exposures is 142.8 mas, we use the scatter of 0.5 mas as a conservative estimate of the uncertainty in this value.

The proper motion of $2 \mathrm{M} 0041$ is $\sim 140 \mathrm{mas} \mathrm{yr}^{-1}$ (Phan Bao $\&$ Bessel 2006), i.e., the primary travels roughly by one full observed separation per year in SE direction. The secondary is sufficiently bright to be visible in archive nIR measurements, if the separation from the primary is large enough. We can use nIR or red observations of the region to search for a second object. Assuming that the secondary would exhibit negligible proper motion, the objects should be separated by $\sim 1.5^{\prime \prime}$ on the 2 MASS images taken in 1999, which would be difficult to detect given the $\sim 3^{\prime \prime} F W H M$ of the 2MASS PSF. On the other hand, ESO.RMAMA plates taken in 1988 should show two objects separated by about one $F W H M$, which is $2.5-3^{\prime \prime}$. We found a second object at the position of $2 \mathrm{M} 0041$ in neither the 2MASS images from 1999 nor the ESO.R-MAMA images from 1988, and we see no signs of an elongated PSF, which would be indicative of a barely resolved secondary object close to the primary $\left(\sim 1^{\prime \prime}\right)$. We conclude that the second object in our images is probably physically bound to the primary. The confirmation of a binary status, however, can only be accomplished by verifying a common proper motion in an exposure taken at a second epoch.

\section{System parameters}

From the new photometry, we can determine the parameters of the two components, and estimate the mass and the orbital period for a given age. The distance to $2 \mathrm{M} 0041$ can be estimated from the difference between absolute and apparent magnitude. For field objects, the absolute magnitude as a function of spectral type was given by Dahn et al. (2002) and Cruz et al. (2003). We can use that estimate to determine a lower limit to the distance that would apply if the objects were field stars. To calculate the distance that would apply if the objects were very young, we scale the absolute magnitude by the radius difference between young and old objects assuming that the temperature does not change significantly with age for a given spectral type. We use the radius-age dependence from Baraffe et al. (1998, 2002).

All parameters and their uncertainties are given in Table 3. Uncertainties are related to the spectral type uncertainty, which has an important effect on the absolute magnitude, and to this we add the uncertainty in $J$ in quadrature. Based on the assumption that $2 \mathrm{M} 0041 \mathrm{~A}$ is a field star, the distance to the system is $24 \mathrm{pc}$. As expected, the new distance is larger than the $17 \mathrm{pc}$ calculated earlier (Faherty et al. 2009), because in that calculation, all the flux measured in $J$ was assumed originate in a single (and cooler) object. We can now use the radius-age relation to estimate the distance for different ages of 2M0041 A (see Reiners 2009). The distances to 2M0041 for ages of 5 and $10 \mathrm{Myr}$ are 71 and $50 \mathrm{pc}$, respectively. To estimate the semi-major axis, we apply a correction factor of 1.26 to account for projection effects (Fischer \& Marcy 1992). The separations between 2M0041 A and B are inferred to be 8.9 and $12.8 \mathrm{AU}$ for a $10 \mathrm{Myr}$ and a $5 \mathrm{Myr}$ binary, respectively.

From the evolutionary tracks of Baraffe et al., we can also estimate the mass of the two components at a given age. With knowledge of both the masses and the separation, we can then estimate the orbital period of the binary assuming that the distance we observe is the true semimajor-axis of the system.

The orbital period that would be derived if the binary were old is $P \approx 22$ yr. This is the lower boundary to the period; the 
Table 3. System parameters for different ages ${ }^{1}$.

\begin{tabular}{ccc|c}
\hline \hline Parameter & \multicolumn{3}{c}{ Value } \\
Age [Myr] & 5 & 10 & MS \\
\hline$d$ [pc] & $71_{-15}^{+29}$ & $50_{-10}^{+21}$ & $24_{-5}^{+10}$ \\
Separation [AU] & $12.8_{-2.7}^{+5.3}$ & $8.9_{-1.9}^{+3.7}$ & $4.3_{-0.9}^{+1.8}$ \\
Mass (A) [ $\left.M_{\text {Jup }}\right]$ & $\sim 25$ & $\sim 30$ & $\sim 95$ \\
Mass (B) [M $\left.M_{\text {Jup }}\right]$ & $\sim 15$ & $\sim 15$ & $\sim 80$ \\
Orbital Period [yr] & $228_{-68}^{+155}$ & $126_{-37}^{+86}$ & $22_{-7}^{+15}$ \\
\hline
\end{tabular}

Notes. ${ }^{(1)}$ The main sequence (MS) case is excluded by the Li detection and given only for reference.

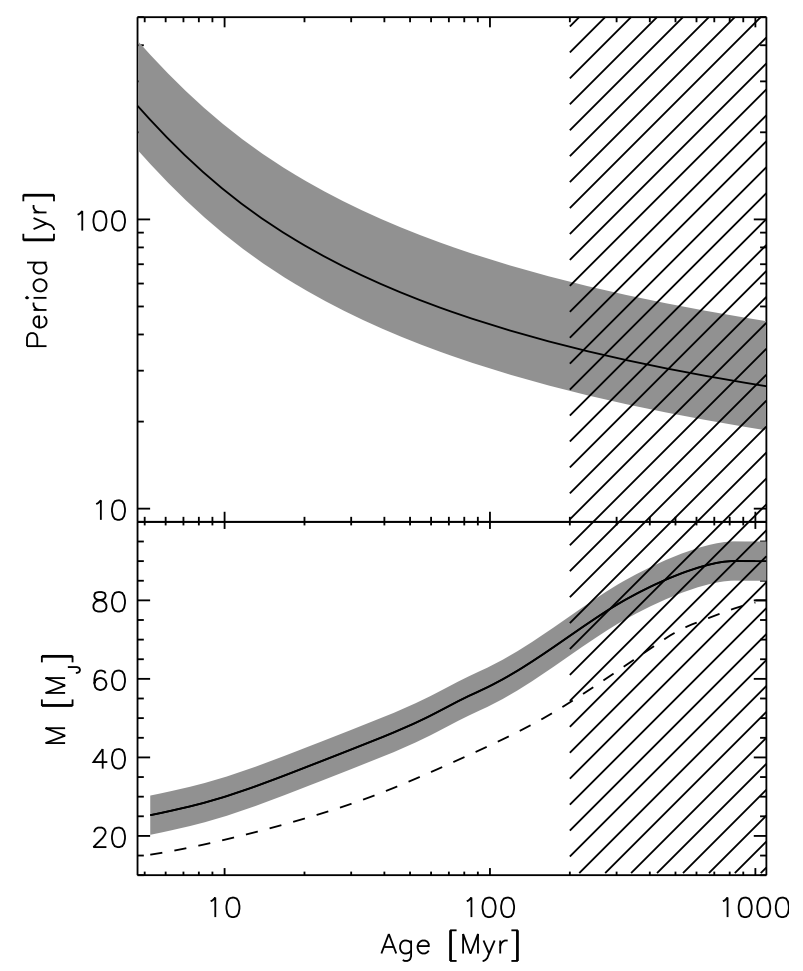

Fig. 2. Top panel: estimated orbital period as a function of age for $2 \mathrm{M} 0041 \mathrm{~A}+\mathrm{B}$ (solid line). The grey region indicates $1 \sigma$ uncertainties (see text). Bottom panel: estimated mass as a function of age. The solid line shows the mass of component $\mathrm{A}$, the dashed line is for component $\mathrm{B}$, uncertainties are shown for component $\mathrm{A}$ only. The hatched area marks the region excluded because of the presence of $\mathrm{Li}$ in the spectrum.

true period must be longer because an age of above a few hundred Myr is excluded by the detection of Li. If 2M0041 had an age of $10 \mathrm{Myr}$, the period would be on the order of $126 \mathrm{yr}$, i.e., a factor of 5 longer. If the system were as young as $5 \mathrm{Myr}$, the period would be about $228 \mathrm{yr}$, i.e., another factor of two longer. We show the estimated period as a function of age in Fig. 2. Using the photometric information gathered about this object, the possible orbital period is found to be a steep function of age.

\section{Summary}

In a NaCo image of the nearby young brown dwarf 2M0041, we have discovered the binarity of this object. The system consists of an M 6.5 primary and a secondary of spectral type M 9.0. We found a separation of 143 mas and derived individual $J, H$, and $K_{\mathrm{s}}$ magnitudes for both components. Flux ratio and colors of both components are consistent, which means that the chances of the secondary being a background object are very small.
The distance, age, mass, and orbital period of the system remain unknown, but we have presented possible solutions as a function of age. The object contains lithium, which means that it is a brown dwarf younger than a few hundred Myr. The detection of $\mathrm{H} \alpha$ and other optical emission lines indicate that the object may be accreting, and that its age may be as low as 10 Myr. For this age, the period is predicted to be on the order of $125 \mathrm{yr}$ at a separation of $\sim 9 \mathrm{AU}$ and a distance of $50 \mathrm{pc}$. Age, semi-major axis, and distance are steep functions of the orbital period. So far, no parallax measurement is available so that the distance to the binary is unknown given that we do not know its age with any great certainty.

Objects of spectral type M9.0 are much fainter at optical wavelengths than M 6.5, so the secondary contributes negligible background continuum to the $\mathrm{H} \alpha$ and Li measurement. On the other hand, the $\mathrm{H} \alpha$ emission seen in the combined spectrum may originate in either of the two components, or both, which would affect the measured accretion rate: if both objects were accreting, the accretion rate per star would be lower, but the shape and strength of $\mathrm{H} \alpha$ would still be indicative of accretion. Alternatively, if the accretion timescale were longer for the secondary, it may be the only accreting object in the system. In that case, the accretion rate would be higher than the value originally derived. An image taken in $\mathrm{H} \alpha$ or even spatially resolved spectroscopy would help resolve this issue.

The discovery of binarity in this young brown dwarf provides an opportunity to directly determine the mass of two young nearby brown dwarfs that may be accreting. Without knowing the distance, the age of the system can be determined from the orbital period. In the next few years, the first estimate of the orbital period should become available. If it were to be as young as a few ten million years, it would be the first object in this mass/age regime for which a direct mass estimate would be available. A measured parallax would give us independent information about the distance and place strong constraints on the system's parameters. This system will be an important benchmark for brown dwarf evolutionary models at young ages.

Acknowledgements. We thank an anonymous referee for a very helpful report. Based on observations made with the European Southern Observatory, PID 383.C0708. This publication has made use of the Very-Low-Mass Binaries Archive housed at http://www.vlmbinaries.org and maintained by Nick Siegler, Chris Gelino, and Adam Burgasser. A.R. acknowledges research funding from the DFG as an Emmy Noether fellow under RE 1664/4-1, A.S. acknowledges financial support from NSF grant AST07-08074.

\section{References}

Baraffe, I., Chabrier, G., Allard, F., \& Hauschildt, P. H. 1998, A\&A, 337, 403 Baraffe, I., Chabrier, G., Allard, F., \& Hauschildt, P. H. 2002, A\&A, 382, 563 Burgasser, A. J., Reid, I. N., Siegler, N., et al. 2007, Protostars and Planets V, ed.

B. Reipurth, D. Jewitt, \& K. Keil (Tucson: University of Arizona Press), 427 Close, L. M., Siegler, N., Freed, M., \& Biller, B. 2003, ApJ, 587, 407

Dahn, C. C., Harris, H. C., Vrba, F. J., et al. 2002, ApJ, 124, 1170

Cruz, K. L., Reid, I. N., Liebert, J., Kirkpatrick, J. D., \& Lowrance, P. J. 2003, AJ, 126, 2421

Diolaiti, E., Bendinelli, O., Bonaccini, D., et al. 2000, A\&AS, 147, 335 Faherty, J. K., Burgasser, A. J., Cruz, K. L., et al. 2009, AJ, 137, 1 Fischer, D. A., \& Marcy, G. W. 1992, ApJ, 396, 178

Hawley, S. L., Covey, K. R., Knapp, G. R., et al. 2002, ApJ, 123, 3409 Lenzen, R., Hartung, M., Brandner, W., et al. 2003, SPIE, 4841, 944 Phan Bao, N., \& Bessel, M. S. 2006, A\&A, 515, 523

Phan Bao, N., Guibert, J., Crifo, F., et al. 2001, A\&A, 380, 590 Reiners, A. 2009, ApJ, 702, L119

Reiners, A., \& Basri, G. 2009, ApJ, 705, 1416

Rousset, G., Lacombe, F., Puget, P., et al. 2003, SPIE 4839, 140 Skrutskie, M. F., Cutri, R. M., Stiening, R., et al. 2006, AJ, 131, 1163 West, A. A., Hawley, S. L., Bochanski, J. J., et al. 2008, ApJ, 135, 785 